\title{
Low-traffic limit and first-passage times for a simple model of the continuous double auction
}

\author{
Enrico Scalas ${ }^{1}$ \\ Department of Mathematics, School of Mathematical and Physical Sciences, University of \\ Sussex, Falmer Campus, BN1 9QH, Brighton, UK and \\ BCAM, Basque Center for Applied Mathematics, Alameda de Mazarredo 14, 48009 Bilbao, \\ Basque Country, Spain \\ Fabio Rapallo \\ DISIT, Università del Piemonte Orientale, viale Teresa Michel 11, 15121 Alessandria, Italy
}

Tijana Radivojević

BCAM, Basque Center for Applied Mathematics, Alameda de Mazarredo 14, 48009 Bilbao, Basque Country, Spain

\begin{abstract}
We consider a simplified model of the continuous double auction where prices are integers varying from 1 to $N$ with limit orders and market orders, but quantity per order limited to a single share. For this model, the order process is equivalent to two $M / M / 1$ queues. We study the behaviour of the auction in the low-traffic limit where limit orders are immediately matched by market orders. In this limit, the distribution of prices can be computed exactly and gives a reasonable approximation of the price distribution when the ratio between the rate of order arrivals and the rate of order executions is below $1 / 2$. This is further confirmed by the analysis of the first-passage time in 1 or $N$.
\end{abstract}

Keywords: Double auction, Markov chains, Queing theory, Low-traffic limit PACS: 89.65.Gh, 02.50.-r

\footnotetext{
Email addresses: e.scalas@sussex.ac.uk (Enrico Scalas), fabio.rapallo@uniupo.it (Fabio Rapallo), tradivojevic@bcamath.org (Tijana Radivojević)

${ }^{1}$ Corresponding author.
} 


\section{Introduction and model description}

Most of the regulated markets in the world implement a trading mechanism known as the continuous double auction to match supply and demand. This mechanism has two sides. On the supply side there are orders to sell and on the demand side there are orders to buy. Hence, the auction is called double. Moreover it occurs in continuous time. Hence, it is called continuous.

To be more specific, in, say, a regulated equity market, a continuous double auction consists of a book for each share where orders to buy (bids) and to sell (asks) are recorded. An order consists of a price at which the seller (buyer) wants to sell (buy), of a quantity of shares they want to sell (buy) and of further specifications, for instance concerning order cancellation. The possible order types are described by market regulations. Orders can be sent directly to the book and, in this case, we have an order-driven market. On the contrary, if only bids and asks sent by market makers and/or specialists are accepted, we have a so-called quote-driven market (see [1] for a classical introduction to the topic and [2] for the new problems related to high-frequency trading). For our purposes, it is important to distinguish between two main order types: Market orders and limit orders. A market order is an order where the seller (buyer) accepts to sell (buy) a certain quantity of shares at the best available price. In other words, the seller (buyer) accepts the best bid (ask) present in the book. When a market order is executed a transaction takes place and shares are exchanged. A limit order is an order to buy a certain quantity of shares at a price not higher than a given value or to sell a certain quantity of shares at a price not lower than a given value. Typically, limit orders are stored in the book until they are totally or partially executed or cancelled after some time if they are not executed.

In recent years, the theory of this auction has gained more and more interest. Despite all the possible complications of real continuous double auctions, their theory can be based on queueing theory. In fact, we can consider the book as a peculiar queue and orders as customers that wait to be served (executed). In 
particular, it has been shown that appropriate models of the double auction can be mapped into a multi-class queue [3], so that its ergodic properties and the limiting invariant distribution can be studied using established techniques [4].

In this paper, we consider a simplified model (see [5] and references therein) where prices take $N$ integer values from 1 to $N$. Only two types of orders are considered: limit orders and market orders. In their turn, limit orders can be either orders to sell a single share at a price not lower than a given amount (asks) or orders to buy a single share at a price not higher than a given amount (bids). In other words, the quantity attached to every limit order is always 1. Among all the asks, the best ask is the smallest ask price, whereas the best bid is the largest bid price. The best bid is always strictly smaller than the best ask. Market orders have also two sides: either they accept the available best bid or the available best ask. For the sake of simplicity, limit ask orders and limit bid orders arrive according to a Poisson process at a rate $\lambda_{a}$ and $\lambda_{b}$, respectively. In the following, we assume symmetry, i.e. $\lambda_{a}=\lambda_{b}=\lambda$. Market orders to buy and market orders to sell arrive separated by durations following the exponential distribution with parameter $\mu_{b}$ and $\mu_{a}$, respectively. Again, symmetry is assumed, namely $\mu_{a}=\mu_{b}=\mu$. Limit ask orders follow the uniform distribution in the interval from $p_{b}+1$ to $p_{b}+n$, where $p_{b}$ is the current best bid and $n \geq 1$ is a parameter of the model. Similarly, limit bid orders are uniformly drawn from the interval $p_{a}-n$ to $p_{a}-1$, where $p_{a}$ is the the current best ask. The accessible states of the auction are limited by the condition $p_{b}<p_{a}$. When $p_{a}$ is between 1 and $n$ ( $p_{b}$ between $N-n+1$ and $N$ ), the bid (respectively ask) interval is restricted correspondingly. For instance, if $p_{a}=1$, bids are impossible. The parameter $n$ acts as a cut-off for price jumps. Eventually, if no orders are present in the auction, the next bid, $b$, is uniformly chosen in the interval $p-n \leq b \leq p$ and the next ask, $a$, is uniformly taken from $p \leq a \leq p+n$, where $p$ is the price of the last trade. Specifying an initial price (the opening auction price) is sufficient to start the auction. A short remark is necessary at this stage: It turns out that order inter-arrival times are not exponentially distributed in real markets (see [6] and references 
therein). This means that the above description in terms of $M / M / 1$ processes should be replaced by a semi-Markov description in terms of $G / G / 1$ processes. However, in this paper, for the sake of simplicity, we will limit our analysis to the Markovian case.

The model described above is essentially the same as in [7] and in [8]. It is a zero intelligence agent-based model [9]. As already mentioned in [5], this version of the model does not use the uniform distribution over $[0, \infty)$ as in [7] and it is not limited to the case in which limit orders arrive only at the best bid/ask price as in [8]. A preliminary discussion of this version was presented in [10]. This model was extensively studied in [11], in the case in which price movements equal one tick. These authors also studied the heavy-traffic limit [12] where functional limit theorems are available leading to diffusion approximations [13, 14, 15].

This class of simple models can also help in clarifying the relationship between market structure and agents behaviour, beyond the zero-intelligence limit $[16,17]$.

In [5], the focus was on the ergodic properties of the model. Based on the fact that the order process is equivalent to two independent $M / M / 1$ queues, it was shown that there are three regimes depending on the value of $\rho=\lambda / \mu$. For $0<\rho<1$, prices are free to fluctuate over the full price range and statistical equilibrium is reached (ergodic regime). For $\rho \geq 1$, the auction is in a nonergodic regime which stabilizes prices. Due to the presence of the parameter $n$, there is an additional transition. If $1 \leq \rho<n$, prices can still fluctuate in a limited range, whereas for $\rho \geq n$, prices eventually fluctuate between two values. This regime cannot be found if one only considers the case $n=1$. It is useful to remark that $\rho$ is the relevant parameter in this model. Given that $\rho$ is the ratio between $\lambda$ and $\mu$, the same value of $\rho$ can be obtained with different values of these rates.

In the following, we further characterize the ergodic regime by considering the so-called low-traffic limit where $\rho \ll 1$. It is a limit where analytic results are available for the price dynamics as discussed below. Moreover, we study the first-passage time of the auction in 1 or in $N$. The study of the first-passage 
time is useful to characterize the price stochastic process. It turns out that this analysis provides useful approximations for the behaviour of the auction model when $\rho<1 / 2$. In the spirit of statistical physics, in the following, we study what happens in the limit of vanishing $\rho$, a situation where an exact solution for the price dynamics is available. In the last section of the paper, we further discuss the meaning of this limit.

It is useful to explicitly show a strict analogy between what we are doing in this paper and what is usually done in statistical physics. Even if the continous double auction is a social construct ${ }^{2}$, once it is put into place, such a construct becomes part of nature, at least temporarily ${ }^{3}$, and prone to scientific investigation. In particular, it becomes possible and interesting to study simplified models of the social construct as it is possible and interesting to study simplified models of natural phenomena such as magnetism. The behavior of the above simplified model is described by a single parameter $\rho$ and, as mentioned above, there is an ergodic transition for $\rho=1$. The ergodic regime is for $0<\rho<1$. The limiting case $\rho \uparrow 1$ was studied in [12]. Here, we study the case $\rho \downarrow 0$. Similarly, simplified models for magnetism were introduced, such as the Ising model. The behaviour of the (e.g. ferromagnetic) Ising model is described by a single parameter $k_{B} T / J$ where $k_{B}$ is Boltzmann's constant, $T$ is absolute temperature and $J>0$ represents spin coupling. In the case of the Ising model in, say, two or three spatial dimensions, there is a phase transition at a finite critical value of $k_{B} T / J$. Especially when exact formulae for the partition function in the thermodynamic limit are not available (as in the case of the three-dimensional Ising model), it is useful to study approximations in the low- $\left(k_{B} T / J \downarrow 0\right)$ and

\footnotetext{
${ }^{2}$ The Oxford Dictionary defines a social construct as a "social phenomenon or convention originating within and cultivated by society or a particular social group, as opposed to existing inherently or naturally".

${ }^{3}$ Without presenting a detailed history of auctions, we can state with some confidence that there has been a long period in human history before the introduction of the continuous double auction. And, perhaps with less confidence, we can state that there will be a more or less remote future without continuous double auction.
} 
high-temperature $\left(k_{B} T / J \uparrow \infty\right)$ limits.

\section{The low-traffic limit}

We give two interpretations of the low-traffic limit $(\rho \ll 1)$. As stated above, $\rho$ is a ratio between $\lambda$ and $\mu$ and our limit can be obtained for constant $\lambda$ and $\mu \rightarrow \infty$. In this case, when limit orders arrive, they are immediately matched by market orders. However, this limit can also be reached by considering a vanishing $\lambda$ and a constant $\mu$. In this case, a limit order is rarely placed in the book and the probability of a new limit order arriving before the execution of the previous one is negligible. This second interpretation is further discussed in Section 4 if the reader has the patience of following us up to there.

Whatever the interpretation, in this limit, it is possible to explicitly write the transition probabilities for the price process and study the price Markov chain for any value of $n$ and $N$. To explain how to proceed, let us assume that the initial price is $p$ as defined in the previous section. Then, the conditional probability of a bid is given by

$$
\mathbb{P}\left(B_{1}=b \mid P_{0}=p\right)=\left\{\begin{array}{lll}
0 & \text { if } & b<p-n \text { or } b>p \\
\frac{1}{p} & \text { if } \quad 1 \leq b \leq p \leq n \\
\frac{1}{n+1} & \text { if } \quad 1 \leq p-n \leq b \leq p .
\end{array}\right.
$$

This bid is immediately accepted and it becomes the next price. A similar set of equations can be written for the asks conditioned to the initial price.

$$
\mathbb{P}\left(A_{1}=a \mid P_{0}=p\right)=\left\{\begin{array}{lll}
0 & \text { if } \quad a<p \text { or } a>p+n \\
\frac{1}{N-p+1} & \text { if } \quad N-n+1 \leq p \leq a \leq N \\
\frac{1}{n+1} & \text { if } \quad p \leq a \leq p+n \leq N .
\end{array}\right.
$$

Both Eq. (1) and (2) are an immediate consequence of the model definition. For a full characterization of the price Markov chain, the distribution of the 
initial price is needed. For instance, if the initial price is chosen uniformly, the probability of an initial price is $1 / N$; if the chain starts from a given price, the probability of this price is 1 and the probabilities of all the other prices are 0 , and so on. In a symmetric auction, for which $\lambda_{a}=\lambda_{b}=\lambda$ and $\mu_{a}=\mu_{b}=\mu$, the probability of a bid arriving is $1 / 2$ and it is equal to the probability of arrival of an ask. Therefore, in the low-traffic regime, the transition probability for prices is given by

$$
P_{p, p^{\prime}}=\mathbb{P}\left(P_{1}=p^{\prime} \mid P_{0}=p\right)=\frac{1}{2} \mathbb{P}\left(A_{1}=p^{\prime} \mid P_{0}=p\right)+\frac{1}{2} \mathbb{P}\left(B_{1}=p^{\prime} \mid P_{0}=p\right) .
$$

Let us now assume that the limit $\rho \ll 1$ is realized by keeping the arrival rate $\lambda$ finite and letting $\mu \gg \lambda$. Then, if $N(t)$ denotes the number of transactions up to time $t$, we have that $N(t)$ is Poisson distributed with parameter $2 \lambda$, given that the auction has two sides (either a bid arrives with rate $\lambda$ or an ask arrives with rate $\lambda$ ). In fact, $N(t)$ is the superposition of two Poisson processes with parameter $\lambda$. In other words, the price process can be seen as an embedded Markov chain characterized by the transition probability (3) subordinated to the Poisson process $N(t)$. Once this remark is made, it is safe to focus on the embedded chain and study its properties. In particular we are interested in the convergence of the price probability. First of all, we notice that after any transactions, the double auction is exactly in the same situation as in the initial case, except for the fact that the price probability varies with time. In other words, the Markov chain defined above is homogeneous. From the study of the transition probability, one can further infer that the Markov chain is irreducible. In fact, it is possible to reach any price from any other price. Moreover, given that the diagonal terms of the Markov transition matrix are all positive, meaning that there is a finite probability for the price not to change at every step, we can conclude that our Markov chain is aperiodic. Being irreducible and aperiodic, our chain has a unique invariant distribution and this is an equilibrium distribution.

In order to illustrate the above findings, let us consider a specific example with $N=10$ prices and $n=2$. In this case, the price transition probability 
matrix in Eq. (3) is

$$
P=\left(\begin{array}{cccccccccc}
4 / 6 & 1 / 6 & 1 / 6 & 0 & 0 & 0 & 0 & 0 & 0 & 0 \\
1 / 4 & 5 / 12 & 1 / 6 & 1 / 6 & 0 & 0 & 0 & 0 & 0 & 0 \\
1 / 6 & 1 / 6 & 1 / 3 & 1 / 6 & 1 / 6 & 0 & 0 & 0 & 0 & 0 \\
0 & 1 / 6 & 1 / 6 & 1 / 3 & 1 / 6 & 1 / 6 & 0 & 0 & 0 & 0 \\
0 & 0 & 1 / 6 & 1 / 6 & 1 / 3 & 1 / 6 & 1 / 6 & 0 & 0 & 0 \\
0 & 0 & 0 & 1 / 6 & 1 / 6 & 1 / 3 & 1 / 6 & 1 / 6 & 0 & 0 \\
0 & 0 & 0 & 0 & 1 / 6 & 1 / 6 & 1 / 3 & 1 / 6 & 1 / 6 & 0 \\
0 & 0 & 0 & 0 & 0 & 1 / 6 & 1 / 6 & 1 / 3 & 1 / 6 & 1 / 6 \\
0 & 0 & 0 & 0 & 0 & 0 & 1 / 6 & 1 / 6 & 5 / 12 & 1 / 4 \\
0 & 0 & 0 & 0 & 0 & 0 & 0 & 1 / 6 & 1 / 6 & 4 / 6
\end{array}\right)
$$

The invariant distribution is obtained by looking for the left eigenvector with unit eigenvalue, namely

$$
\pi P=\pi
$$

which, in the case of (4) gives

$$
\pi=(0.1171,0.0895,0.1,0.0961,0.0974,0.0974,0.0961,0.1,0.0895,0.1171) .
$$

In Appendix A, we present a general algorithm to find the invariant distribution of prices. For $n=1$, there is a remarkable result. In fact, in this case, the transition matrix is a symmetric, doubly-stochastic matrix. Since $P \mathbf{1}=\mathbf{1}$ and $\mathbf{1}^{T} P=\mathbf{1}^{T}$ (because row sums and column sums are 1 ), then

$$
\frac{1}{N} \mathbf{1}^{T} P=\frac{1}{N} \mathbf{1}^{T}
$$

and the uniform distribution is the invariant distribution for the Markov chain.

Always for purpose of illustration, in Figure 1, we plot the low-traffic limit price distribution for the case $N=50, n=5$ and we compare it with the frequency with which states appear after equilibration in a Monte Carlo simulation of the chain after $10^{6}$ iterations for $\rho=10^{-4}$. In this case $10^{6}$ iterations are already sufficient to show that the agreement between the low-traffic-limit approximation and the result of Monte Carlo simulations is good. It is striking 


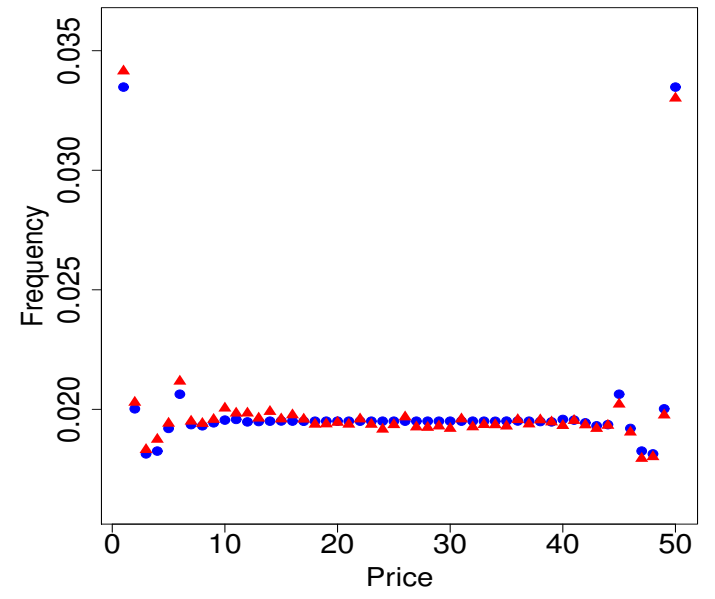

Figure 1: (Color online) Equilibrium price distribution in the low-traffic limit in the case $N=50, n=5$ (circles). The triangles denote the price frequency for a Monte Carlo simulation of the double auction with $\rho=10^{-4}$ after $10^{6}$ steps.

to observe that the price distribution in the low-traffic limit is still a reasonable approximation when $\rho=0.3$ as shown in Figure 2. The approximation breaks down for $\rho \geq 0.5$ as shown in Figure 3 for the case $\rho=0.9$. This behaviour of the price distributions leads to a different behaviour for first-passage times at the boundary prices. In fact, for $\rho>0.5$ and $n>1$ the residence time of the systems close to the boundaries becomes negligible as shown by Figure 3, leading to an increase of the value of the average first-passage time.

\section{First-passage times}

In this section we focus on first-passage times. Given a double auction with $N$ possible prices, labeled with the integers $1, \ldots, N$, we fix the initial price at the median point $\lfloor(N+1) / 2\rfloor$, where $\lfloor\cdot\rfloor$ denotes the floor operator. We study the random variable $T$ : The first-passage time at 1 or at $N$; for this reason, our problem belongs to the class of two-barrier problems, and, for simplicity, we will assume that the price walk is symmetric. The behaviour of the firstpassage time distribution has been studied in several similar problems, both 


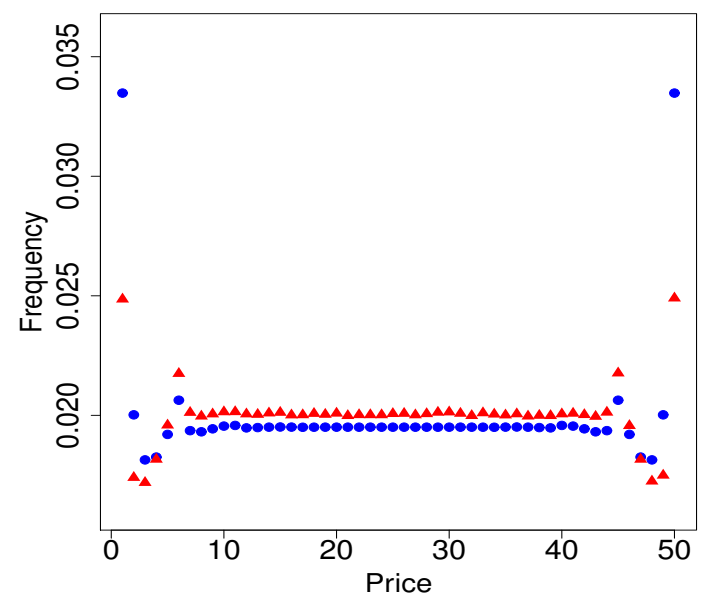

Figure 2: (Color online) Comparison between the equilibrium price distribution in the lowtraffic limit in the case $N=50, n=5$ (circles) and a a Monte Carlo simulation (triangles) of the double auction with $\rho=0.3$ after $10^{9}$ steps.

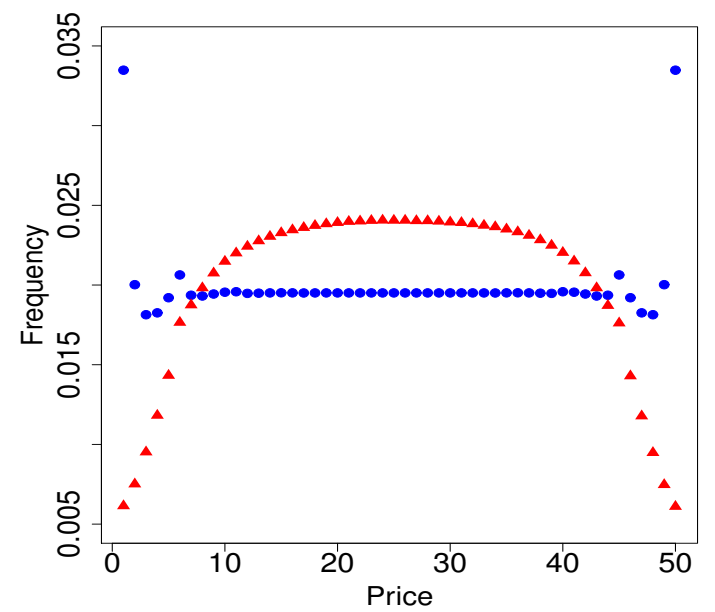

Figure 3: (Color online) Comparison between the equilibrium price distribution in the lowtraffic limit in the case $N=50, n=5$ (circles) and a a Monte Carlo simulation (triangles) of the double auction with $\rho=0.9$ after $10^{9}$ steps. 
$\mathrm{N}=\mathbf{1 0}$

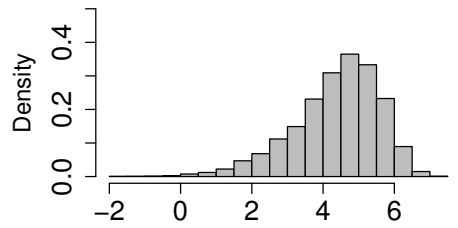

$\mathrm{N}=70$

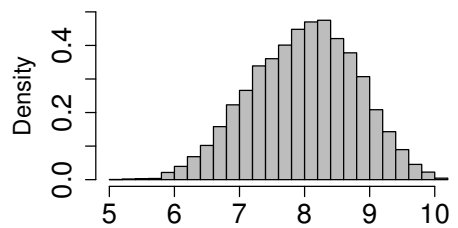

$\mathrm{N}=40$

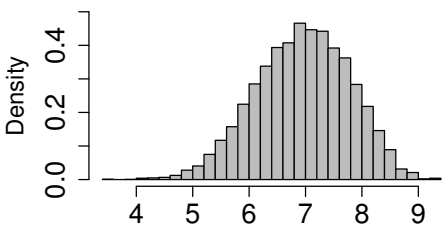

$\mathrm{N}=100$

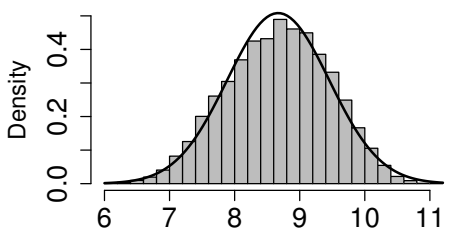

Figure 4: Distribution of $\log (T)$ for $n=5$ fixed and $\rho=0.02$.

with theoretical results on the exact or asymptotic distribution, and through simulation studies, see e.g. [18] and [19].

Here, we present the results of a simulation study to investigate the main features of the distribution of $\log (T)$. In particular, we compare such a distribution with the theoretical distribution derived under the low-traffic assumption. Since the distribution of $T$ is highly skewed with a fat upper tail, as shown in figures, all the plots reported here refer to the distribution of its natural log$\operatorname{arithm} \log (T)$. For sake of simplicity, we have performed all comparisons in this section with the parameter $\mu=1$ fixed. With this assumption, we get the low-traffic limit if $\rho=\lambda \ll 1$.

In Figure 4 the histograms of $\log (T)$ for $n=5$ and for $\rho=0.02$ are displayed for 4 different values of $N$, namely $N=10,40,70,100$. One can observe that the shape of the distribution is skewed for small values of $N$, while it approaches a Gaussian distribution in the case $N=100$ (the best-fit normal curve is plotted together with the histogram). The simulations have been implemented in $\mathrm{R}$ [20], and all histograms in this study are based on 10,000 Monte-Carlo replicates. 
$\mathrm{N}=\mathbf{1 0}$

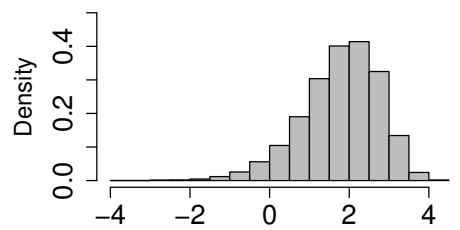

$\mathrm{N}=70$

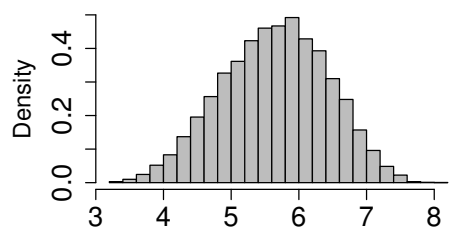

$\mathrm{N}=\mathbf{4 0}$

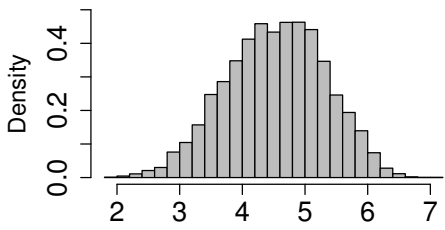

$\mathrm{N}=100$

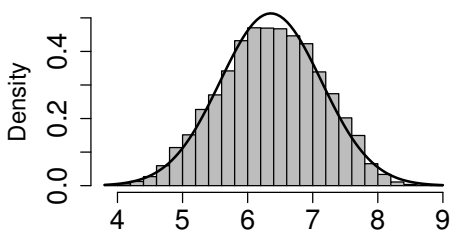

Figure 5: Distribution of $\log (T)$ for for $n=5$ fixed and $\rho=0.5$.

Figure 5 refers to the same settings as above, but with the ratio fixed at $\rho=0.5$. The distribution of $\log (T)$ has almost the same shape for $\rho=0.5$ and for $\rho=0.02$. In agreement with the conclusions of the previous section based on the limiting distribution, this fact suggests that the study of the lowtraffic approximation is worth to be analyzed also for relatively large values of $\rho$, namely for $\rho$ up to 0.5 . Nevertheless, the different location and scale parameters in the histograms in Figures 4 and 5 highlight that a reasonable approximation of the first-passage time must use the ratio $\rho$ to control the location and scale of the approximation. We will consider this task further in this section.

Finally, in Figure 6, the means of $\log (T)$ as a function of $\rho$ under various choices of $N$ ( $n=5$ fixed $)$ are displayed. A minimum occur between $\rho=0.4$ and $\rho=0.5$ except for the first experimental setting $(N=10)$.

To complete the simulation study, we have compared the distribution of $T$ with an approximation suggested by the results of the previous section. Basically, we adapt here a known formula for a discrete two-barrier problem. Such formula gives the distribution of the number of price changes needed to reach the 

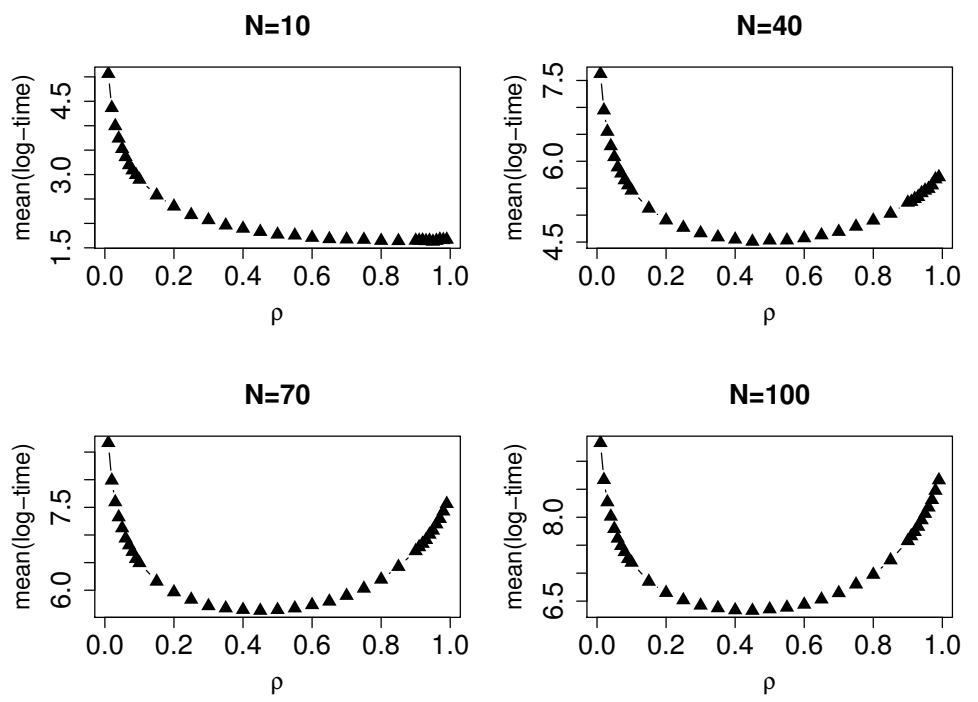

Figure 6: Mean of $\log (T)$ for $n=5$ and $N=10,40,70,100$.

boundary. Then, the parameter $\rho$ controls the proportion of orders leading to a price change, and therefore it defines the discrete time distribution of the number of orders needed to reach the boundary. Remember that in the low-traffic limit when limit orders arrive, they are immediately matched by market orders. Finally, we add suitable exponential distributions to switch to the continuous framework of our model. To avoid problems in some formulae, we assume here $N$ to be odd, so that $(N+1) / 2$ is always integer. We have limited our study to the case $n=1$ in order to avoid further technicalities in the formulae and to capture the major features of the model. The approximation is built up in three steps, as detailed below:

- First, consider a discrete first-passage time $\widetilde{T}^{(d)}$ in a simple symmetric random walk with two reflecting barriers and discrete \pm 1 steps, whose 
distribution is

$$
\begin{aligned}
\mathbb{P}\left(\widetilde{T}^{(d)}=\tilde{h}\right)= & \frac{2}{N-1} \times \\
& \sum_{k=1}^{N-2}(-1)^{k+1} \sin \left(\frac{k \pi}{N-1}\right) \cos ^{\tilde{h}-1}\left(\frac{k \pi}{N-1}\right) \sin \left(\frac{k \pi}{2}\right)
\end{aligned}
$$

for $\tilde{h} \geq 1$. The above distribution can be found in [21] and is extensively discussed with several generalizations in [22].

- The rate of arrival of limit orders over all orders is $\lambda /(\lambda+\mu)=\rho /(\rho+1)$, and in the low-traffic approximation all limit orders arrive when the book is empty. A limit order to buy (resp. to sell) fixes the price at the old price $p$ or at $p+1$ (resp. $p-1$ ) with probability $1 / 2$ each. Therefore, at any given time, the price changes with rate $\rho /\{2(\rho+1)\}$. Thus, given $\widetilde{T}^{(d)}=\tilde{h}$, consider a Negative Binomial variable $N B$ with parameters $\tilde{h}$ and $\rho /\{2(\rho+1)\}$, and define $T^{(d)}=N B+1$. The variable $T^{(d)}$ is again a discrete random variable and it counts the number of events after the change of price is actually performed;

- The interarrival time between two consecutive events follows an exponential distribution with mean $2 \mu(1+\rho)$. Therefore, given $T^{(d)}=h$, the firstpassage time is approximated by a random variable following a Gamma distribution with parameters $h$ and $1 /\{2 \mu(1+\rho)\}$.

In conclusion, the distribution of the first-passage time can be approximated by a suitable mixture $T^{(a)}$ of Gamma distributions, whose parameters are computed according to the formula in Eq. (6) for the discrete case. Notice that in the previous construction the low-traffic hypothesis is used only when we assume the book empty when a limit order arrives.

To show that this approximation works well for small values of $\rho$, we have plotted in Figure 7 the empirical Monte Carlo cumulative distribution function $(\mathrm{ECDF})$ of $\log (T)$ and the distribution of $\log \left(T^{(a)}\right)$ for 4 different values of $\rho$ in the case $N=11$. Also in these simulations we have considered only the case $\mu=1$. 

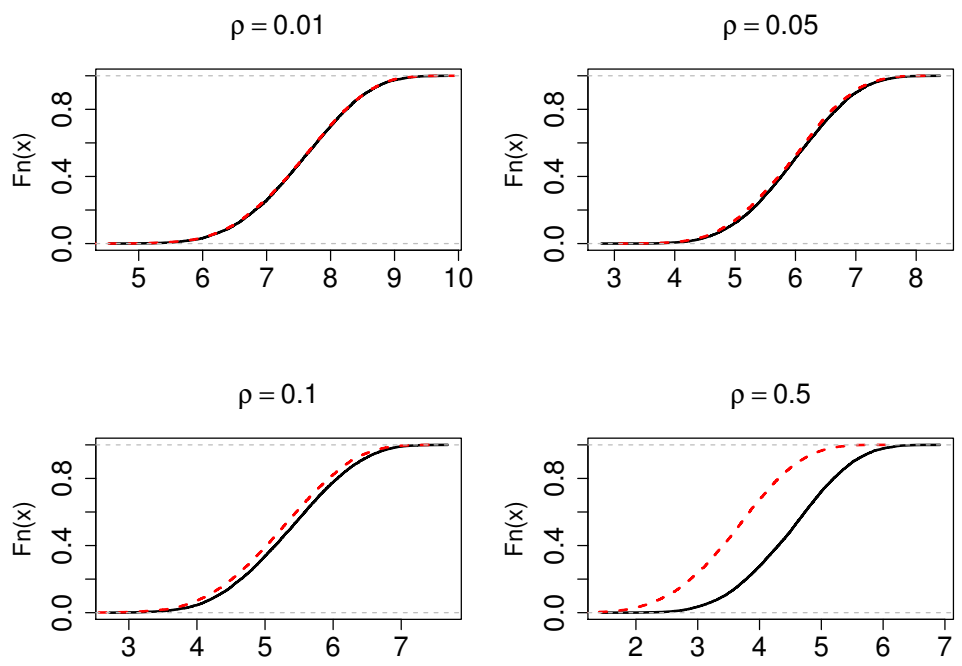

Figure 7: (Color online) ECDFs of $\log (T)$ (solid, in black) and its low-traffic approximation $\log \left(T^{(a)}\right)$ (dashed, in red) for $N=11, n=1$.

We can observe in Figure 7 that for $\rho=0.01, \rho=0.05$ the simulated distribution and its theoretical approximation are nearly identical (the $p$-value of the Kolmogorov-Smirnov based on 10,000 Monte Carlo replicates is 0.7212 for $\rho=0.01$ and 0.0116 for $\rho=0.05)$. When $\rho=0.1$ the two distributions show some discrepancies, while in the last scenario $(\rho=0.5)$ the approximation fails. The low-traffic approximation $T^{(a)}$ tends to underestimate the distribution of $T$. This behaviour is observed also in the $\rho=0.1$ case, but it is clearer in the $\rho=0.5$ case, as expected.

As a further analysis, in Figure 8, we have plotted the Kullback-Leibler divergence between the Monte Carlo empirical distribution of $\log (T)$ and its low-traffic approximation as a function of $\rho$, for $\rho$ ranging from 0.01 to 0.5 , in accordance with the values used in Figure 7 . The settings are the same, namely $N=11$ and $n=1$. From Figure 8 we can see that the increase of the divergence for values of $\rho$ up to about 0.15 is rather slow, while it is rapidly growing for values of $\rho$ greater than 0.2 . 


\begin{tabular}{cc|ccccc|cccc} 
& & \multicolumn{5}{|c|}{$\rho=0.01$} & \multicolumn{3}{c}{$\rho=0.02$} \\
$N$ & $n$ & $\bar{T}$ & $\mu_{T}$ & $\Delta \%$ & & $N$ & $n$ & $\bar{T}$ & $\mu_{T}$ & $\Delta \%$ \\
\hline 10 & 5 & 273.17 & 275.67 & -0.91 & & 10 & 5 & 136.58 & 137.76 & -0.85 \\
40 & 5 & 2787.28 & 2821.84 & -1.22 & 40 & 5 & 1393.64 & 1433.86 & -2.80 \\
40 & 10 & 1176.20 & 1197.19 & -1.75 & 40 & 10 & 588.10 & 604.54 & -2.72 \\
80 & 5 & 9939.48 & 9888.86 & +0.51 & 80 & 5 & 4969.74 & 5181.18 & -4.08 \\
80 & 20 & 1598.81 & 1617.58 & -1.16 & 80 & 20 & 799.41 & 824.12 & -3.00 \\
100 & 5 & 15151.93 & 15183.58 & -0.21 & & 100 & 5 & 7575.97 & 7807.49 & -2.97 \\
100 & 25 & 1763.36 & 1768.67 & -0.30 & 100 & 25 & 881.68 & 903.18 & -2.38 \\
\hline
\end{tabular}

\begin{tabular}{cc|ccccc|cccc} 
& & \multicolumn{5}{|c|}{$\rho=0.05$} & & \multicolumn{3}{c}{$\rho=0.10$} \\
$N$ & $n$ & $\bar{T}$ & $\mu_{T}$ & $\Delta \%$ & $N$ & $n$ & $\bar{T}$ & $\mu_{T}$ & $\Delta \%$ \\
\hline 10 & 5 & 54.63 & 56.44 & -3.20 & & 10 & 5 & 27.32 & 29.47 & -7.31 \\
40 & 5 & 557.46 & 598.03 & -6.78 & 40 & 5 & 278.73 & 319.18 & -12.67 \\
40 & 10 & 235.24 & 248.15 & -5.20 & 40 & 10 & 117.62 & 132.71 & -11.37 \\
80 & 5 & 1987.90 & 2164.12 & -8.14 & 80 & 5 & 993.95 & 1159.32 & -14.26 \\
80 & 20 & 319.76 & 342.19 & -6.55 & 80 & 20 & 159.88 & 182.86 & -12.57 \\
100 & 5 & 3030.39 & 3267.66 & -7.26 & 100 & 5 & 1515.19 & 1780.02 & -14.88 \\
100 & 25 & 352.67 & 369.58 & -4.58 & 100 & 25 & 176.34 & 198.62 & -11.22 \\
\hline
\end{tabular}

\begin{tabular}{cc|ccc} 
& & \multicolumn{3}{|c}{$\rho=0.50$} \\
$N$ & $n$ & $\bar{T}$ & $\mu_{T}$ & $\Delta \%$ \\
\hline 10 & 5 & 5.46 & 8.72 & -37.35 \\
40 & 5 & 55.75 & 124.99 & -55.40 \\
40 & 10 & 23.52 & 45.54 & -48.35 \\
80 & 5 & 198.79 & 495.32 & -59.87 \\
80 & 20 & 31.98 & 62.29 & -48.66 \\
100 & 5 & 303.04 & 769.96 & -60.64 \\
100 & 25 & 35.27 & 70.41 & -49.91 \\
\hline
\end{tabular}

Table 1: Average times in the real settings and the corresponding low-traffic approximation for different values of $N, n$ and $\rho$. 


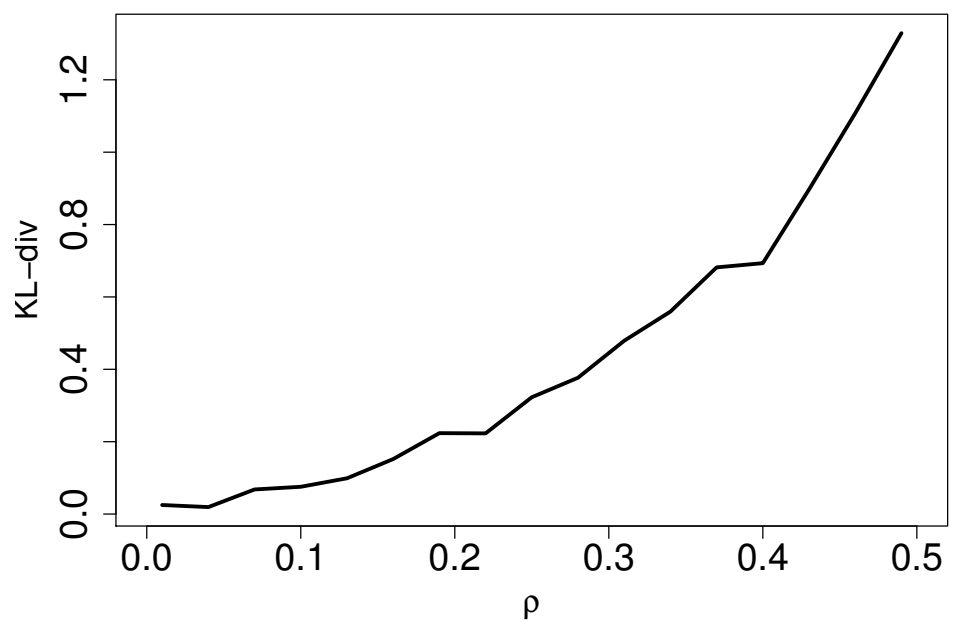

Figure 8: Kullback-Leibler divergence between the empirical distribution of $\log (T)$ and its low-traffic approximation for $N=11, n=1$.

When $n>1$ a formula like that in Eq. (6) is no longer available. However, we can analyze the low-traffic approximation by studying the expected values of the distributions. In fact, the expected value of the first-passage time $\mu_{T}^{(d)}=\mathbb{E}\left(T^{(d)}\right)$ of the discrete chain in the low-traffic approximation can be computed through the linear system

$$
\left(I-P_{2, N-1}\right) x=1
$$

where $P_{2, N-1}$ is the transition matrix restricted to the transient states, $I$ is the $(N-2) \times(N-2)$ identity matrix, and 1 is a column vector of 1 with dimension $N-2$ (see e.g. [23] for details). Then, the mean time in the continuous setting $\mu_{T}$ is obtained by scaling $\mu_{T}^{(d)}$ by a factor $1 /(2 \rho)$, following the same reasoning as above. In Table 1 , the means $\bar{T}$ of the Monte Carlo simulations and the theoretical expected value under the low-traffic approximation $\mu_{T}$ are given for several settings. For $\rho$ up to 0.05 the approximation works well, and the relative error is less than $10 \%$ in all settings, while for $\rho=0.1$ and $\rho=0.5$ the differences become relevant, especially in the latter case. 


\section{Discussion}

In this paper, we characterized the ergodic regime of a simple model for the continuous double auction in the low-traffic limit $\rho \ll 1$. In this limit, the price distribution can be exactly derived for any value of the model parameters $n$ and $N$. Explicit numerical procedures to find the price distributions are given in Appendix A. We also showed that these results give a reasonable approximation of the auction behaviour for $\rho<1 / 2$.

We further studied the first-passage time $T$ in 1 or $N$ using Monte Carlo simulations. We noticed that the low-traffic limit approximation for $T$ reasonably works for $\rho \ll 1 / 2$ in this case.

At the beginning of Sect. 2, we described the low-traffic limit of vanishing $\rho$ as a limit in which $\lambda$ is constant and $\mu \rightarrow \infty$. In such a situation, when a limit order is placed in the book, it is immediately executed. Alternatively, this limit can also be reached by considering a vanishing $\lambda$ and a constant $\mu$. In this case, a limit order is occasionally placed in the book and no new limit order arrives before its execution. In other words, we have the following chain of events. Let us assume a limit order is sent to the book, but the market is so illiquid that this limit order stays there for some time. No other limit orders are sent before someone accepts the offer. Then the book remains empty for some time before a new limit order comes and the process starts again. In these conditions, the bid-ask spread is not defined as only a single bid or or a single ask is in the market when the book is not empty before a new market order arrives. One should also keep in mind that the new limit orders are not placed at an arbitrary price. In fact, as written in Section 1, if no orders are present in the book, the next bid is uniformly chosen in the interval $p-n \leq b \leq p$ and the next ask is uniformly drawn from $p \leq a \leq p+n$, where $p$ is the last traded price. The sequence of events described above is characteristic of an extreme illiquid market, it might not happen in reality, but it is logically and physically possible. Moreover, for $n \ll N$, the price of the last trade acts as an anchor for the value of the next limit order in the empty book. 
Finally, there are several open questions we would like to answer. A natural extension of this simple model is its semi-Markov version in which non exponential distributions for waiting times between events are introduced. In such an extension, the behavior of the embedded chain does not change, but the mixing time of the chain changes. A particularly interesting case is when the distribution of waiting times is heavy-tailed with infinite mean. This is linked to recent results of ours on semi-Markov graph dynamics [24, 25]. A further research direction worth exploring is considering non-independent processes for limit and market orders.

Even if there are several possible interesting mathematical developments, there are also promising lines of research coupling experimental and behavioral finance with well-understood probabilistic models for market microsructure as suggested in the introduction $[16,17]$. This could be done along the lines of [26]. In that paper, results of an agent-based model of the double auction were compared with results of experiments with human participants in well-defined settings.

\section{Acknowledgements}

T. Radivojević and E. Scalas wish to thank J. Anselmi for useful discussion. E. Scalas thanks N. Georgiou for discussion on multi-class queues. T. Radivojević would like to thank the financial support from the project of the Spanish Ministry of Economy and Competitiveness MINECO with reference MTM201676329-R (AEI/FEDER, EU), and from the Basque Government ELKARTEK Programme, grant KK-2016/0002. This research is also supported by the Basque Government through the BERC 2014-2017 program and by the MINECO: BCAM Severo Ochoa accreditation SEV-2013-0323.

\section{Author contribution statement}

All authors have equally contributed to the completion of the manuscript. 


\section{Appendix A}

In this appendix, we present a general algorithm to find the invariant distribution for the price Markov chain. First, we observe that, in general, the transition probability matrix is a stochastic band matrix, and the following block choice seems to be convenient in the example of eq. (4):

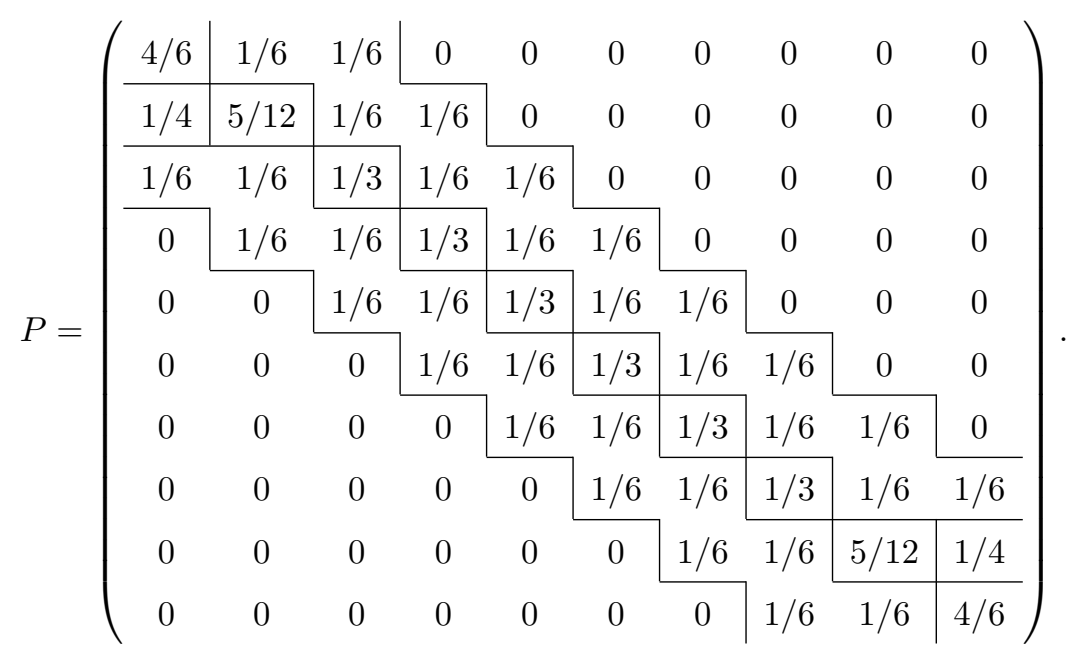

To better see how this generalizes, let us consider the structure of the matrix for $N=10$ and $n=2$, once more

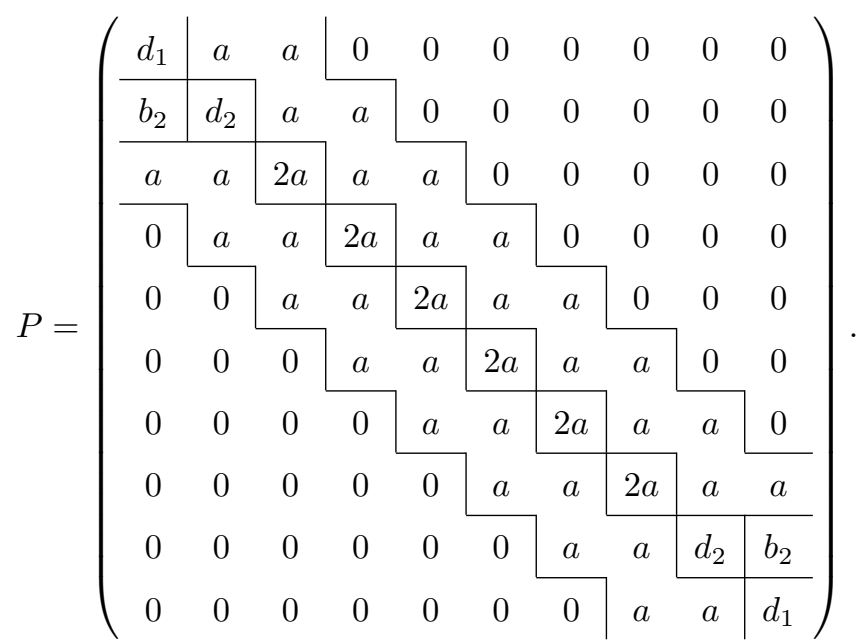


For $N=10, n=3$, instead, we have

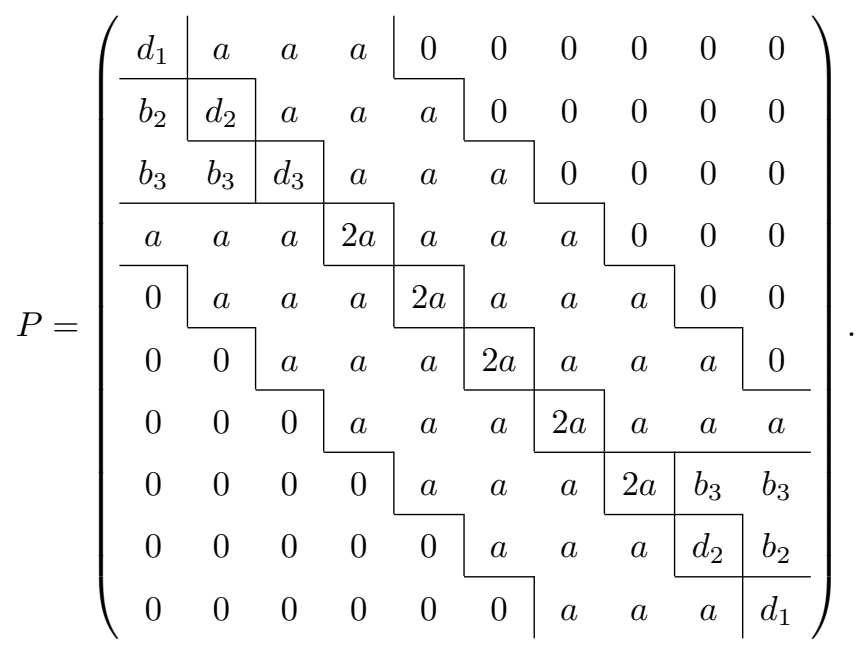

and so on, where:

$$
a=\frac{1}{2(n+1)} ; \quad d_{i}=\frac{1}{2 i}+a ; \quad b_{i}=\frac{1}{2 i}
$$

as a consequence of Eq. (3) in the paper and

$$
\begin{gathered}
d_{1}+n a=1 \\
b_{2}+d_{2}+n a=1 \\
b_{3}+b_{3}+d_{3}+n a=1 \\
\vdots \\
(n-1) b_{n}+d_{n}+n a=1 \\
2 n a+2 a=1
\end{gathered}
$$

as a consequence of the properties of the transition matrix.

The linear system of equations whose solution is the invariant distribution 
from Eq. (5) in the paper is:

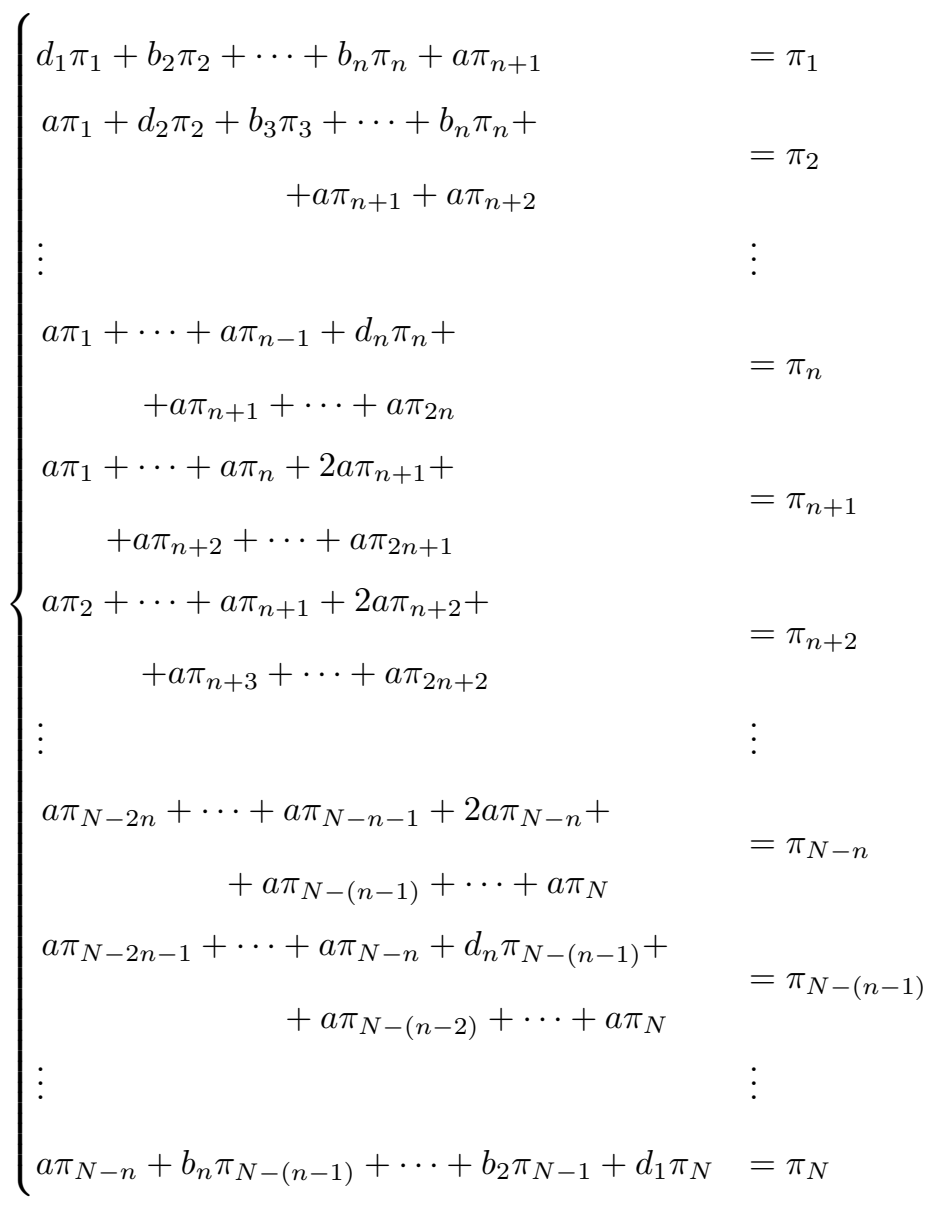

with the additional equation:

$$
\sum_{i=1}^{N} \pi_{i}=1 .
$$

A possible numerical solver for the system (A.1) given $N$ and $n$, and written in MATLAB is:

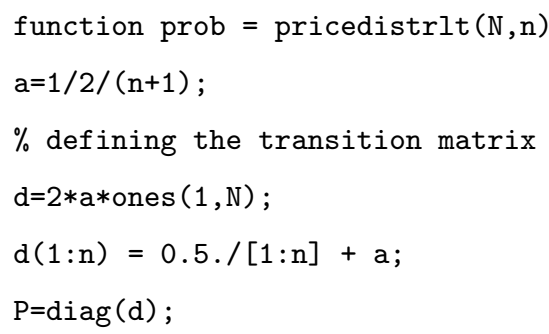




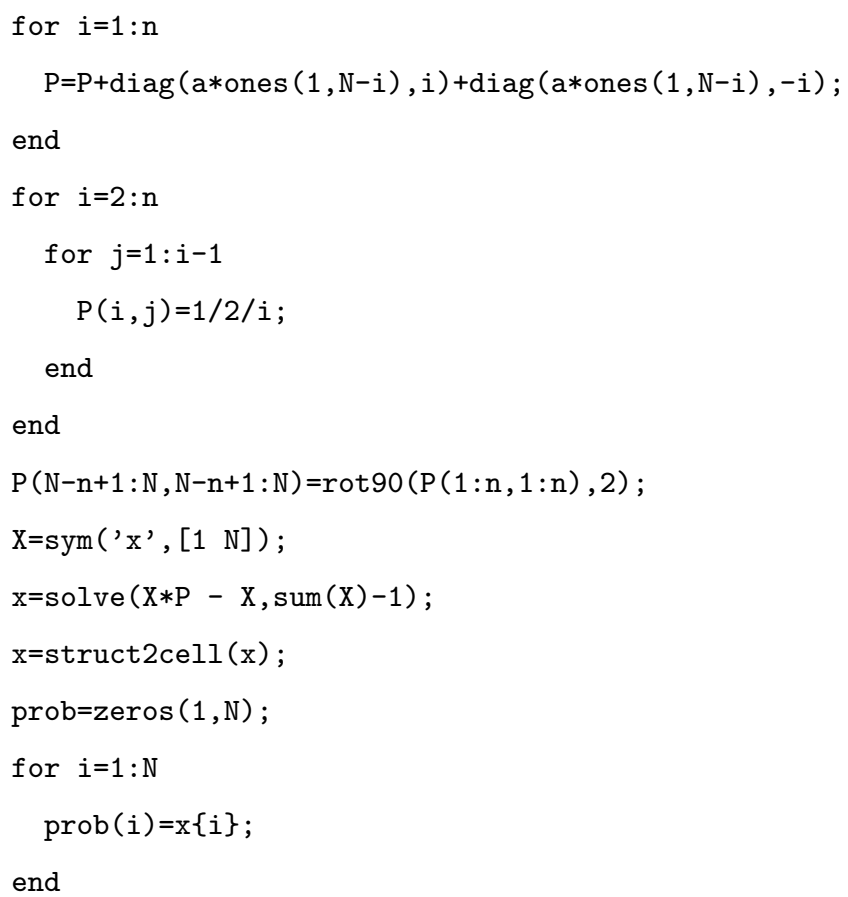

The above function requires the Symbolic Math Toolbox in MATLAB. Another possibility is to solve (A.1) using the Matlab function eig after defining the transition matrix in the following way:

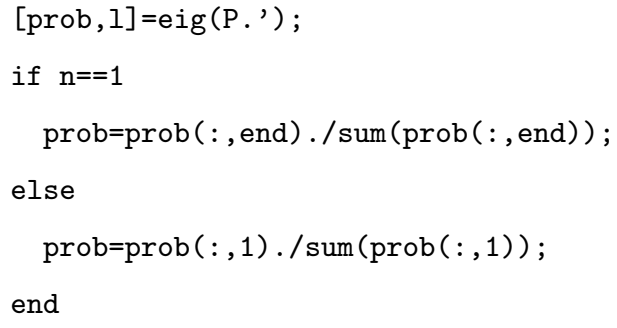

This option is much faster, but less accurate.

\section{References}

[1] M. O’Hara, Market Microstructure Theory, Wiley, 1998.

[2] M. O'Hara, High frequency market microstructure, J. Financ. Econ 116 (2) 
(2015) 257-270. doi:https://doi.org/10.1016/j.jfineco.2015.01. 003.

[3] J. Blanchet, X. Chen, Continuous-time modeling of bid-ask spread and price dynamics in limit order books (2013).

URL arXiv: 1310.1103 [q-fin.TR]

[4] P. A. Ferrari, J. B. Martin, Multiclass Hammersley-Aldous-Diaconis process and multiclass-customer queues, Ann. Inst. H. Poincaré 45 (1) (2009) 250-265. doi:doi:10.1214/08-AIHP168.

[5] T. Radivojević, J. Anselmi, E. Scalas, Ergodic transition in a simple model of the continuous double auction, PLoS ONE 9 (2:e88095) (2014) 1-5. doi: https://doi.org/10.1371/journal.pone.0088095.

[6] E. Scalas, T. Kaizoji, M. Kirchler, J. Huber, A. Tedeschi, Waiting times between orders and trades in double-auction markets, Physica A 366 (2006) 463-471. doi:https://doi.org/10.1016/j.physa.2005.09.047.

[7] E. Smith, J. D. Farmer, L. Gillemot, S. Krishnamurthy, Statistical theory of the continuous double auction, Quant. Financ. 3 (2002) 481-514.

[8] S. Zuo, M. Li, K. Kishimoto, M. Endo, A queuing model for a continuous double-auction trading system, in: 22nd Australasian Finance and Banking Conference, 2009. doi:http://dx.doi.org/10.2139/ssrn.1456762.

[9] D. K. Gode, S. Sunder, Allocative efficiency of markets with zerointelligence traders: Market as a partial substitute for individual rationality, J. Polit. Econ. 101 (1993) 119-137.

[10] T. Radivojević, J. Anselmi, E. Scalas, A stylized model for the continuous double auction, in: A. Teglio, S. Alfarano, E. Camacho-Cuena, M. Ginés-Vilar (Eds.), Managing Market Complexity: The Approach of Artificial Economics, Vol. 662 of Lecture Notes in Economics and Mathematical Systems, Springer, Berlin, Heidelberg, 2012, pp. 115-125. doi: 10.1007/978-3-642-31301-1_10. 
[11] R. Cont, A. de Larrard, Price dynamics in a Markovian limit order market, SIAM J. Finan. Math. 4 (2013) 1-25. doi:10.1137/110856605.

[12] R. Cont, A. de Larrard, Order book dynamics in liquid markets: Limit theorems and diffusion approximations, working paper (2012).

URL http://ssrn. com/abstract=1757861

[13] J. F. Kingman, The single server queue in heavy traffic, Proc. Cambridge Philos. Soc. 57 (1961) 902-904.

[14] J. F. Kingman, On queues in heavy traffic, J. Roy. Statist. Soc. Ser. B 24 (1962) 383-392.

[15] W. Whitt, Stochastic-Process Limits, Springer, 2002.

[16] G. Bottazzi, G. Dosi, I. Rebesco, Institutional architectures and behavioral ecologies in the dynamics of financial markets, J. Math. Econ. 41 (2005) 197-228. doi:https://doi.org/10.1016/j.jmateco.2004.02.006.

[17] M. Anufriev, V. Panchenko, Asset prices, traders' behavior and market design, J. Econ. Dyn. Control 33 (9) (2009) 1073-1090. doi:https:// doi.org/10.1016/j.jedc.2008.09.008.

[18] L. Bondesson, A characterization of first passage time distributions for random walks, Stoch. Proc. Appl. 39 (1991) 81-88. doi:https://doi. org/10.1016/0304-4149(91)90033-9.

[19] T. Antal, S. Redner, Escape of a uniform random walk from an interval, J. Stat. Phys. 123 (2006) 1129-1144. doi:10.1007/s10955-006-9139-2.

[20] R. C. Team, R: A language and environment for statistical computing (2014).

URL http://www.R-project.org/

[21] W. Feller, An Introduction to Probability Theory and Its Applications, Vol. 1, John Wiley \& Sons, 1968. 
[22] A. Carlsund, First passage time for random walks and birth-and-death processes with sign depending transition probabilities, working Paper Royal Institute of Technology, Stockholm (2000).

[23] J. G. Kemeny, J. L. Snell, Finite Markov Chains, Springer-Verlag, 1976.

[24] M. Raberto, F. Rapallo, E. Scalas, Semi-Markov graph dynamics, PLoS ONE 6 (8:e23370) (2011) 1-13. doi:https://doi.org/10.1371/journal. pone. 0023370 .

[25] N. Georgiou, I. Kiss, E. Scalas, Solvable non-Markovian dynamic network, Phys. Rev. E 92 (042801). doi:https://doi.org/10.1103/PhysRevE. 92. 042801.

[26] B. Tóth, E. Scalas, J. Huber, M. Kirchler, The value of information in a multi-agent market model: The luck of the uninformed, Eur. Phys. J. B 55 (1) (2007) 115-120. doi:DOI:10.1140/epjb/e2007-00046-2. 